\title{
Backscattering in carbon nanotubes : role of quantum interference effects
}

\author{
Stephan Roche ${ }^{\ddagger}$, François Triozon ${ }^{\dagger}$ and Angel Rubio * \\ ${ }^{\ddagger}$ Commissariat à l'Énergie Atomique, DRFMC/SPSMS, Grenoble, France. \\ ${ }^{\dagger}$ LEPES-CNRS, avenue des Martyrs BP166, 38042 Grenoble, France. \\ * Departamento de Física de Materiales, Facultad de Ciencias Químicas, Universidad del Pais \\ Vasco/Euskal Herriko Unibertsitatea, Apdo. 1072, 20018 San Sebastián/Donostia, Basque \\ Country, Spain.
}

\begin{abstract}
The backscattering contribution to the conductivity, irrelevant for metallic single-walled carbon nanotubes, is proved to become much significant for doped semiconducting systems, in agreement with experiments. In the case of multi-walled nanotubes, the intershell coupling is further shown to enhance the contribution of backscattering for "metallic" double-walled, whereas it remains insignificant for "metallic/semiconducting" double-walled systems.
\end{abstract}


Single-walled carbon nanotubes (SWNTs) can be either metallic of semiconducting depending on their helicities, i.e. how the graphene sheet is rolled upl. For weak uniform disorder, the application of the Fermi golden rule for metallic SWNTs have demonstrated $\mu m$ long mean free path ${ }^{2}$ in agreement with experiments, clearly pointing towards ballistic transport国国.

Notwithstanding, quantum transport in SWNTs is richer than in the one-dimensional chain, given the implication of additional symmetries of electronic eigenstates associated to the circumferential helicity. This has been widely illustrated through the theoretical study of conduction upon introduction of single defects such as vacancies, impurities or topological defects 6 . In particular, the absence of backscattering was demonstrated for single impurity with long range potential in metallic tubest and stepwise reduction of conductance was inferred from multiple scattering on a few lattice impurities胭. Resonant electronic scattering by defects was recently confirmed experimentally?

On the other hand, electrostatically or chemically doped semiconducting SWNTs have been reported to behave as diffusive conductors with short mean free paths, several orders of magnitude lower than the ones of reported structurally equivalent metallic SWNT5 50 . 10 . In all these experiments, the mean free path $\left(l_{e}\right)$ is deduced from the measured conductance using $G \sim\left(e^{2} / h\right) \ell_{e} / L_{\text {tube }}\left(L_{\text {tube }}\right.$ the length of the SWNT) and values range from $2 n m 10$ to about $\sim 30 \mathrm{~nm}$ 目. Upon doping, the position of the chemical potential (Fermi energy) with respect to the charge neutrality point is shifted downward (hole-doping) or upward (electron-doping) and hence may come closer to a Van-Hove singularity. This may result in a factor $\sqrt{\left\langle v^{2}\right\rangle}$ much smaller than the typical $v_{F}$ deduced from the metallic SWNTs. Moreover, quantum interference effects (QIE) responsable for localization in 1D-systems need to be clarified in the context of carbon nanotubes. Indeed, on multi-walled carbon nanotubes, Bachtold et al. 12 have reported negative magnetoresistance and Aharonov-Bohm oscillations, consistent with the manifestation of quantum interferences in the weak localization regime. This experiment was interpreted by assuming a current predominantly carried in the outermost shell (taken metallic), and mean free path and coherence lengths were deduced from 
conventional theory. It is surprising that quantum interferences that have been described for the two-dimensional propagation (with many more conducting channels), still account properly for the behavior of a single metallic nanotube shell, which only presents two conducting channels at the charge neutrality point. In that perspective, the debate of ballistid 13 against diffusive 12 conduction in MWNTs is a great issue of concern.

All these considerations can be addressed by rewriting the conductance as $\left(e^{2} \sqrt{\left\langle v^{2}\right\rangle} \tau_{e}-\mid\right.$ $\delta \sigma \mid) / L_{\text {tube }}\left(\tau_{e}\right.$ the mean free time of eigenstates given by the Fermi golden rule), and by evaluating properly the contribution of backscattering. This quantum correction $(|\delta \sigma|)$ to the Bloch-Boltzmann conductivity $\left(\sigma_{B B}\right)$, is related to the probability of return to the origin of electronic wavepackets, that is connected to the Participation Ratio (PR), an energy dependent quantity which measures the "spreading" of the electron eigenstates commonly used to address QIE in weak or strong localization regimes 15,16 . For an eigenstate $\psi(E)$ described by its $N$ coefficients $\psi_{i}(E)$ in a tight-binding basis set, the PRs read

$$
P R(E)=\left(\sum_{i}^{N}\left|\psi_{i}(E)\right|^{2}\right)^{2} / \sum_{i}^{N}\left|\psi_{i}(E)\right|^{4}
$$

and it can be shown that an average of the probability of return to the origin in real space is equivalent to an average of the inverse PR on the spectral bandwidth. Accordingly, the amplitude of quantum correction to the electronic conductivity can be estimated as 17

$$
\delta \sigma / \sigma_{B B} \sim P R^{-1}(\text { for } N \rightarrow \infty)
$$

Eigenstates characterized by a linear scaling in $N$ are uniformly extended and associated with a vanishing contribution of QIE, i.e. $\delta \sigma / \sigma_{B B} \rightarrow 0$. Instead, localized states are related to strong contributions of QIE, i.e. $\delta \sigma / \sigma_{B B} \simeq 1$, whereas scaling laws $P R(N)=N^{\alpha}$, with $0<\alpha<1$, indicate the relative strength of QIE.

In this letter we present detailed calculations of the PRs for different tubes, addressing the role of disorder and intertube coupling in the transport properties of nanotubes. We use the standard and reliable one-electron tight binding model including intertube interactions 
(for MWNTs) fitted to ab-initio calculations19. Disorder is included by a random modulation of onsite energies within the range $[-W / 2, W / 2](W=0.054,0.135,0.98 \mathrm{eV})$ that simulate chemical substitutions. The mean free path associated for a given disorder is deduced from $\ell_{e} \sim\left(\gamma_{0} / W\right)^{2} d_{n t},\left(d_{n t}\right.$ is the nanotube diameter, $\gamma_{0}=2.67 \mathrm{eV}$ the hopping between carbon sites)20.

Effect of disorder in SWNTs.-The density of states (DoS) together with the PRs of several metallic chiral and achiral SWNTs are reported on Fig.1 (results are nearly identical for $W=0.054 \mathrm{eV}$ and $0.135 \mathrm{eV}$ ). For Fermi energies at the charge neutrality point, $P R=$ $N$ for armchair and achiral tubes, whereas $P R \sim 2 N / 3$ for zig-zag SWNTs. An energy dependence relation between the position of Van-Hove singularities and the amplitude of PRs is also found. For the Bloch states of zigzag and armchair tubes (the central sub-band of armchairs excepted), there is a degeneracy due to mirror-inversion symmetry. Each value of the wavevector is associated to two Bloch states with the same energy, but with an opposite phase variation along the circumference : $\psi_{n}^{+}=\exp \left(i k \theta_{n} / 2 \pi\right)$ and $\psi_{n}^{-}=\exp \left(-i k \theta_{n} / 2 \pi\right)$, where $k>0$ is a positive integer and $\theta_{n}$ is the polar angle of the site $n$ located on a given ring. By a linear combination of $\psi^{+}$and $\psi^{-}$, a Bloch state with any PR value between $2 N / 3$ and $N$ can be constructed. The lowest value is given by the combination $\left(\psi^{+}+\psi^{-}\right) / \sqrt{2}$, which is a "standing wave" along the circumference : $\psi_{n}^{+}+\psi_{n}^{-}=2 \cos \left(k \theta_{n} / 2 \pi\right)$, leading to $P R=$ $N\left(\left\langle\cos ^{2}\left(k \theta_{n} / 2 \pi\right)\right\rangle\right)^{2} /\left\langle\cos ^{4}\left(k \theta_{n} / 2 \pi\right)\right\rangle=2 N / 3$. Due to this uncertainty, the PR is thus not a well-defined quantity for degenerate eigenstates. But a small amount of disorder is enough to split degeneracy and the PRs shown in Fig.1 become meaningful. For nondegenerate eigenstates (as found in chiral metallic SWNTs and armchair SWNTs close to Fermi energy), perturbation theory applies, so that the PRs are not much reduced by disorder and remain close to $N$. Instead, for degenerate states (zig-zag metallic SWNTs), disorder favors standing waves, i.e. states with a real wavefunction, because the hamiltonian is real and symmetric. Thereby the PRs are close to $2 N / 3$, which explains the general behavior shown in Fig.1. Values much smaller than $2 N / 3$ are attributed to standing waves along the tube axis $z$, obtained by mixing $k_{z}$ and $-k_{z}$ Bloch states close to the Van-Hove singularities. At the 
charge neutrality point of the metallic SWNT $(6,6)$, the eigenstates are basically insensitive to disorder and follow the linear scaling expected for fully extended states (inset Fig.2). This confirms that the effect of small disorder is purely marginal in metallic SWNTs close to Fermi energy 2 日.

On the contrary, a stronger contribution of QIE for doped semiconducting SWNTs is demonstrated by the scaling behavior of PRs as reported on Fig.2 (main frame). The chemical potential of the $(7,5)$ semiconducting tube has been upshifted by assuming a typical dopant concentration of a few percent of carbon sites (within the rigid band approximation). Departure from linear scaling is obtained $\left(P R=N^{\alpha}\right)$ with an increasing contribution of QIE with disorder strength $(\alpha \sim 0.98,0.95$ for $W=0.054 \mathrm{eV}$ and $0.136 \mathrm{eV}$ respectively). For a larger disorder strength $(W=0.98 \mathrm{eV})$, that corresponds to a mean free path $\ell_{e} \simeq 20 \mathrm{~nm}$, the saturation of the PR provides an approximate localization length of $\xi \simeq 40 \mathrm{~nm}$. From the Thouless argument, it is believed that $\xi \sim 4 \times \ell_{e}$ for a metallic shell that provides two conducting channels at the charge neutrality point220. Hence, for doped semiconducting tubes, the mixing between quantum channels induced by substitutional disorder, results in an enhanced contribution of QIE, and lower localization lengths. This demonstrates that the conduction mechanism in the outermost shell of a MWNT depends on the position of the chemical potential12 13. To complete this argument, we add the effect of intertube coupling as it promotes charge transfer between shells in MWNTs.

Effect of disorder and intershell coupling in commensurate MWNTs.-The characteristic sensitivity to disorder of QIE in MWNTs is illustrated on Fig.3 for small disorder $(W=$ $0.054 \mathrm{eV})$. For the double-walled "metallic" armchair tube $(6,6) @(11,11)$, the average PR close to the charge neutrality point is roughly half the value for the isolated armchair tube, for same disorder parameter. According to the previous discussion, the $|\delta \sigma|$ contribution is now not negligible and contribute to a reduction of the conductivity (confirmed by the scaling analysis of PR-not shown here). Such effect is similar in double-walled "metallic" zig-zag tube, but it is reduced when the outer shell is semiconducting. It can be understood from the fact that, at the charge neutrality point, the states of such MWNTs are mainly weighted in 
the inner metallic shell, so less sensitive to the delocalization induced by intershell coupling, and less affected by disorder as in metallic SWNTs. Hence, MWNTs consisting of $\sim 2 / 3$ of semiconducting shells remain long ballistic conductors at charge neutrality point 13.

Acknowledgments: Financial support from NAMITECH [ERBFMRX-CT96-0067(DG12MITH)], DGES (PB98-0345), COMELCAN( HPRN-CT-2000-00128), JCyL (VA28/99) and C are acknowledged. 


\section{REFERENCES}

$\ddagger$ Corresponding author, E-mail: sroche@cea.fr

${ }^{1}$ R. Saito, G. Dresselhaus, and M. S. Dresselhaus, Physical Properties of Carbon Nanotubes (Imperial College Press, London, 1998).

${ }^{2}$ C.T. White and T. N. Todorov, Nature 393, 240 (1998)

${ }^{3}$ M. Bockrath et al., Science 275, 1922 (1997).

${ }^{4}$ A. Bachtold et al., Phys. Rev. Lett. 84, 6082 (2000).

${ }^{5}$ R. Martel et al., Appl. Phys. Lett. 73, 2447 (1998). S. J. Tans et al. ,Nature 393, 49 (1998).

${ }^{6}$ V.H. Crespi, AM.L. Cohen and A. Rubio, Phys. Rev. Lett.79, 2093 (1997). M. P. Anantram and T. R. Govindan, Phys. Rev. B 58, 4882 (1998). T. Kostyrko et al., Phys. Rev. B 59, 3241 (1999).

${ }^{7}$ T. Ando, T. Nakanishi and R. Saito, J. Phys. Soc. Jpn. 67, 2857(1998).

${ }^{8}$ H.J. Choi et al., Phys. Rev. Lett. 84, 2917 (2000).

${ }^{9}$ M. Bockrath et al., Science 291, 283 (2001).

${ }^{10}$ P.L. McEuen et al., Phys. Rev. Lett. 83, 5098 (1999).

${ }^{11}$ M. Krüger et al., to appear in Appl. Phys. Lett.

12 A. Bachtold et al., Nature 397, 673 (1999).

${ }^{13}$ S. Frank et al., Science 280, 1744 (1998).

14 T.W. Ebessen et al., Nature 382, 54 (1996).

${ }^{15}$ F. Wegner, Z. Phys. B., 36, 209 (1980). M. Schreiber, Phys. Rev. B 31, R6146 (1985).

16 J.P. Carini, K.A. Muttalib and S. R. Nagel, Phys. Rev. Lett. 53, 102 (1984). 
${ }^{17}|\delta \sigma|$ is related to $\frac{2 e^{2}}{h} 2 D \int_{0}^{\infty} d t \mathcal{P}(r=0, t) e^{-t / \tau_{\varphi}}\left(1-e^{-t / \tau_{e}}\right) 18$, with $D=v_{F}^{2} \tau_{e}, \tau_{e}=\ell_{e} / v_{F}$ the mean free time, and $\tau_{\varphi}$ the inelastic time which limitates the contribution of the probability of return to the origin $\mathcal{P}(r=0, t)$. Such quantity is given by $|\langle r|\exp (-i H t / \hbar)| r\rangle|^{2}$.

${ }^{18}$ D.K. Ferry and S.M. Goodnick in Transport in nanostructures, (Cambridge University Press, U.S.A. 1997)

${ }^{19}$ R. Saito, G. Dresselhaus, and M. S. Dresselhaus, J. Appl. Phys. 73, 494 (1993). J.C. Charlier and J.P. Michenaud, Phys. Rev. Lett. 70, 1858 (1993).

${ }^{20}$ T. Kostyrko et al., Phys. Rev. B 60, 10735 (1999). S. Roche et al., Phys. Rev. B 62, 16092 (2000). 


\section{Figure captions:}

Figure 1: PRs (solid lines) and TDoS (dashed lines) for metallic armchair $(6,6)$, zigzag $(18,0)$ and chiral $(9,6)$ tubes. PRs are normalized to the number $N$ of atoms, and have been averaged over a few disorder configurations $(W=0.054 \mathrm{eV})$. TDoS are given in arbitrary units.

Figure 2: Comparison of the scaling of the PRs for metallic $(6,6)$ (inset) and doped semiconducting $(7,5)$ single-walled nanotube (for which the chemical potential lies nearby a Van-Hove singularity, $E_{F} / \gamma_{0} \sim 0.333$ upshifted with respect to the charge neutrality point). The dashed lines indicate the linear scaling $(P R=N)$. Comparison is made for two values of disorder strength $W=0.054 \mathrm{eV}$ (open circles) and $0.136 \mathrm{eV}$ (filled circles). The third disorder strength $W=0.98 \mathrm{eV}$ (filled diamonds) taken for $(7,5)$ at the same Fermi energy leads to $\ell_{e} \sim 20 \mathrm{~nm}$.

Figure 3: PRs for different double-walled tubes : "metallic" armchair $(6,6) @(11,11)$, zigzag $(9,0) @(18,0)$, and "metallic/semiconducting" chiral $(9,6) @(15,10)$ nanotubes. 


\section{FIGURES}

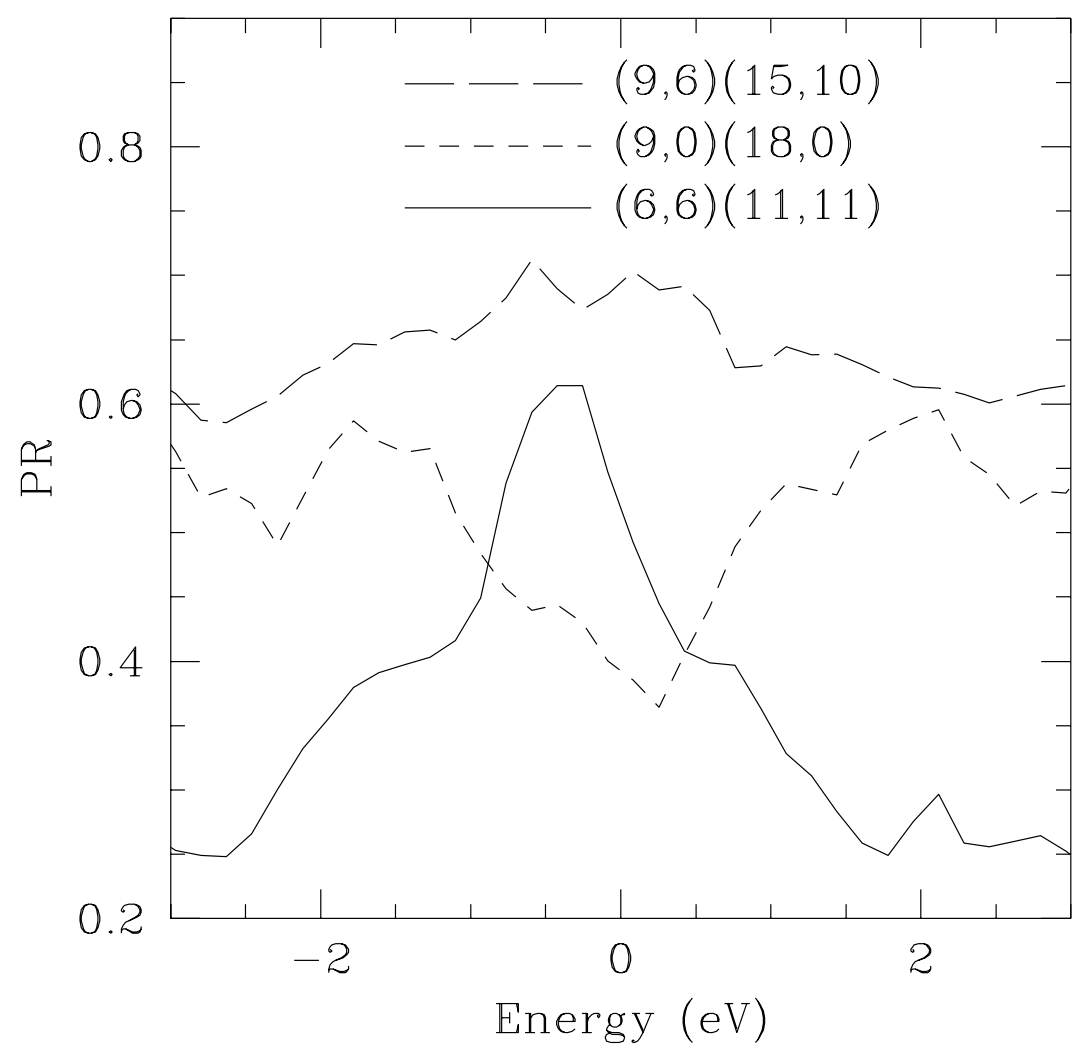




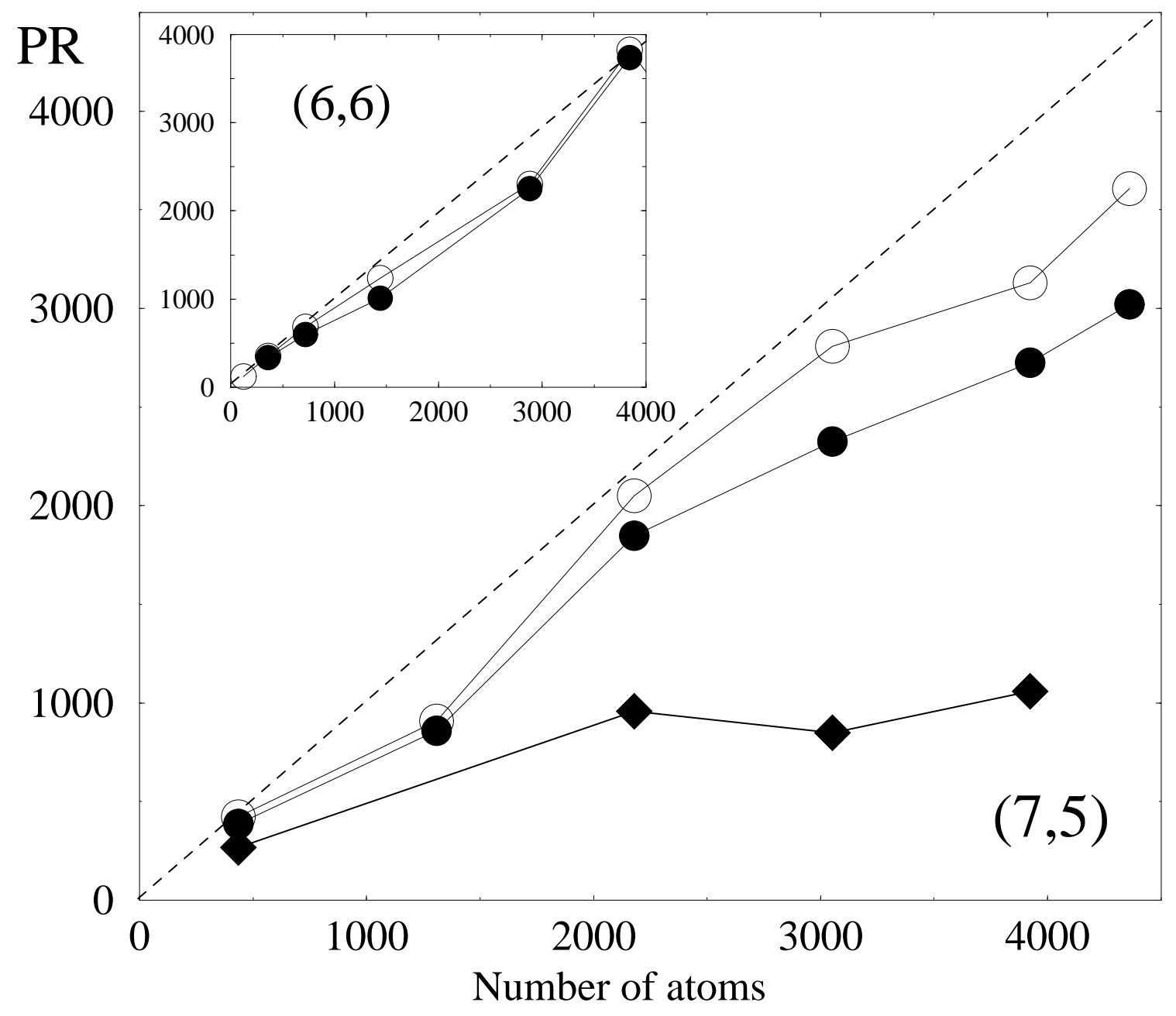




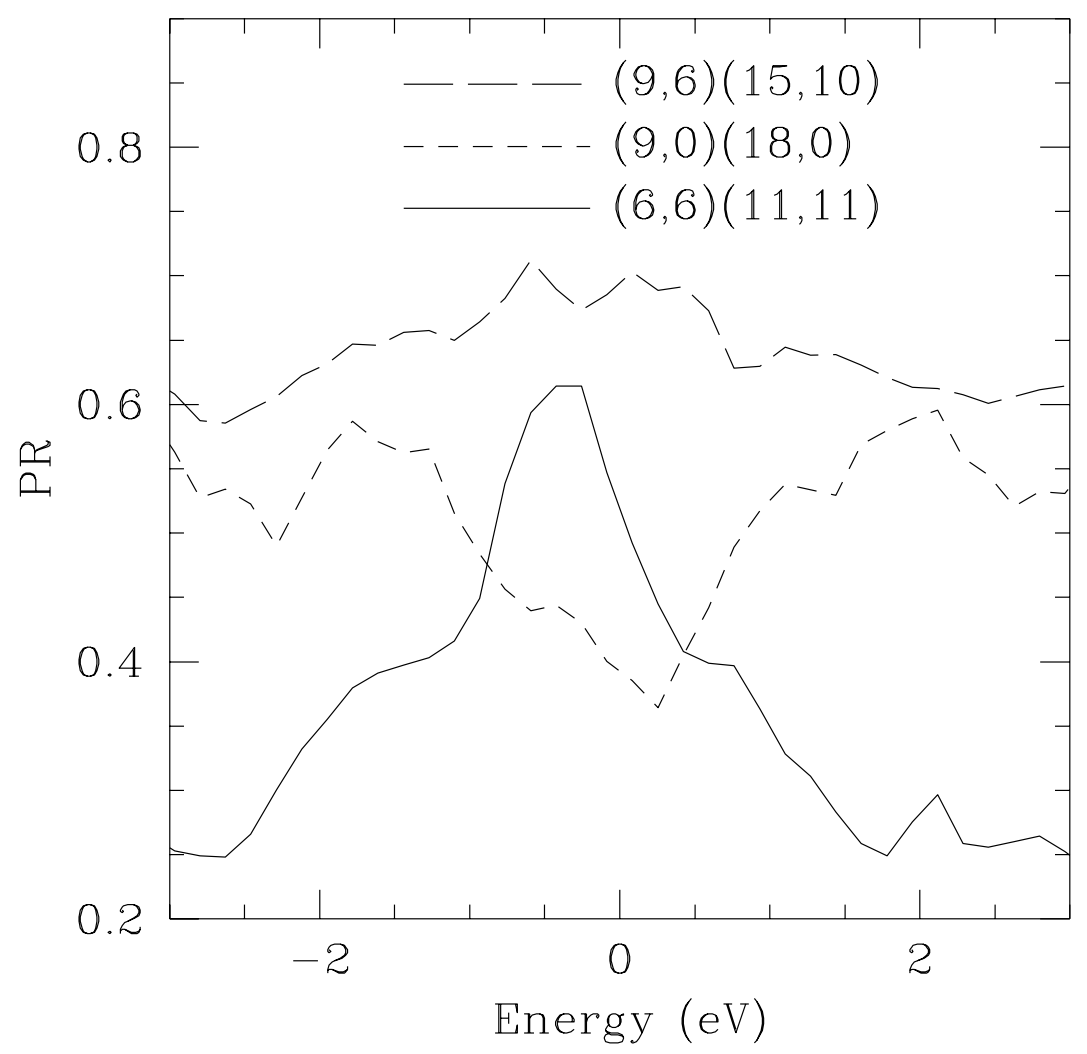

1-12 


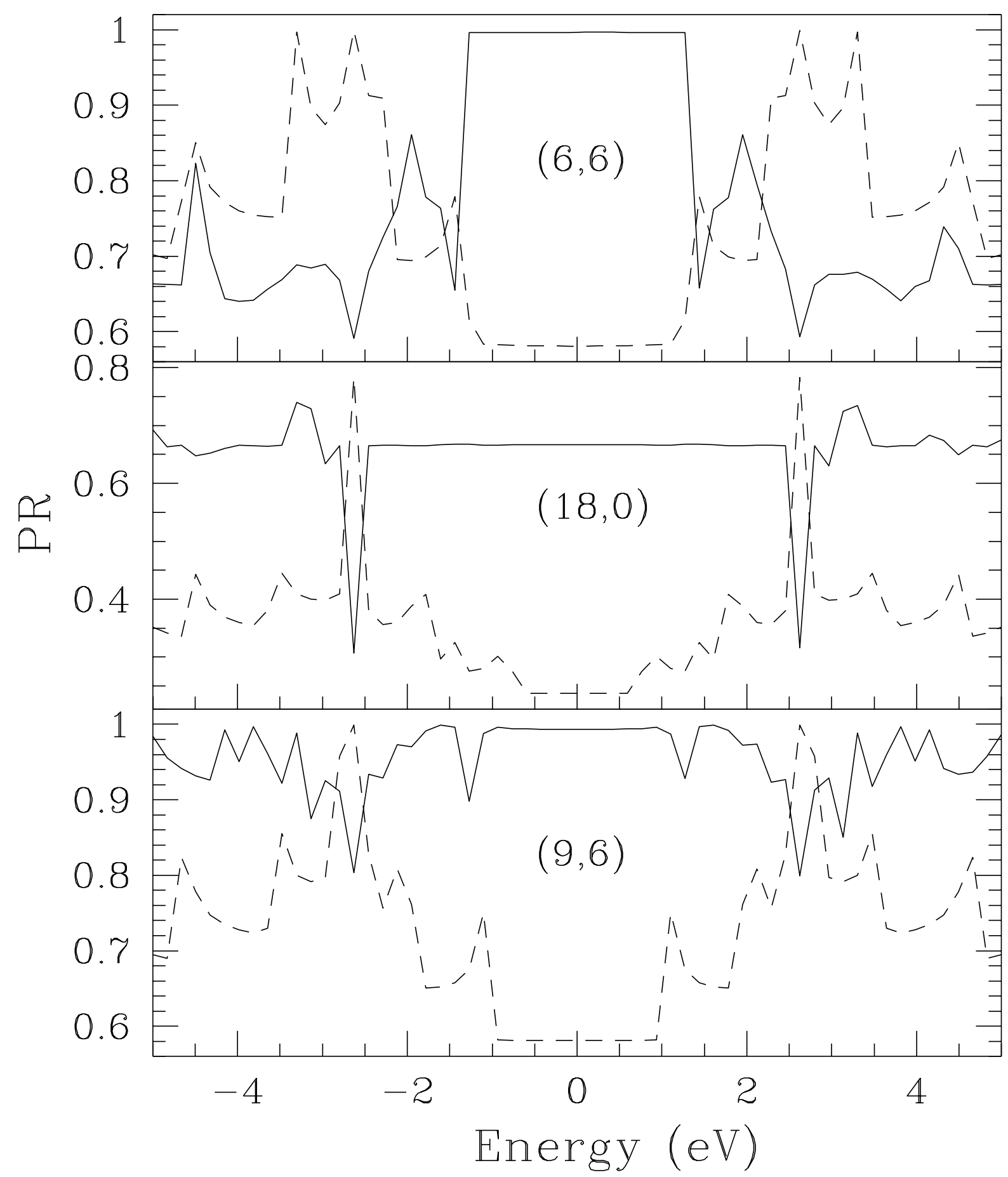

